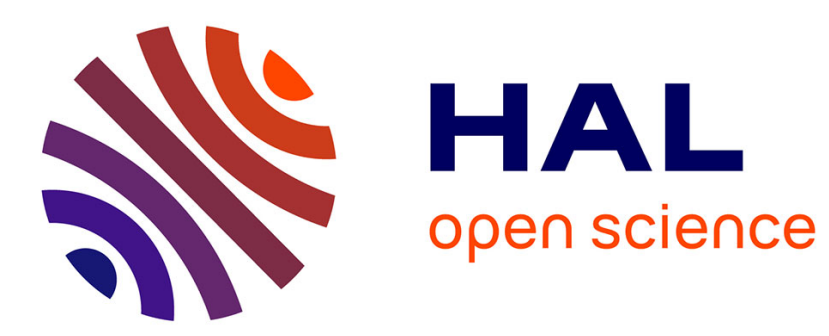

\title{
Elastic-plastic analysis of the peel test for ductile thin film presenting a saturation of the yield stress
}

\author{
E. Simlissi, M. Martiny, Sébastien Mercier, S. Bahi, L. Bodin
}

\section{To cite this version:}

E. Simlissi, M. Martiny, Sébastien Mercier, S. Bahi, L. Bodin. Elastic-plastic analysis of the peel test for ductile thin film presenting a saturation of the yield stress. International Journal of Fracture, 2019, 220 (1), pp.1-16. 10.1007/s10704-019-00393-7 . hal-02351987

\section{HAL Id: hal-02351987 \\ https://hal.univ-lorraine.fr/hal-02351987}

Submitted on 6 Nov 2019

HAL is a multi-disciplinary open access archive for the deposit and dissemination of scientific research documents, whether they are published or not. The documents may come from teaching and research institutions in France or abroad, or from public or private research centers.
L'archive ouverte pluridisciplinaire HAL, est destinée au dépôt et à la diffusion de documents scientifiques de niveau recherche, publiés ou non, émanant des établissements d'enseignement et de recherche français ou étrangers, des laboratoires publics ou privés. 


\title{
Elastic-plastic analysis of the peel test for ductile
}

\section{thin film presenting a saturation of the yield}

\author{
stress.
}

E. Simlissi ${ }^{a, b}$ and M. Martiny ${ }^{a, *}$ and S. Mercier ${ }^{a}$ and S. Bahi ${ }^{a}$ and L. Bodin ${ }^{b}$

${ }^{a}$ Université de Lorraine, CNRS, Arts et Métiers ParisTech, LEM3, F-57000 Metz, France

${ }^{b}$ Cimulec, rue charles Picard, 57365 Ennery, France

\begin{abstract}
The paper investigates the peel test of an elastic-plastic film on an elastic substrate. The case of a film material presenting a saturation of the yield stress is considered. Based on earlier approaches of the literature, see for instance Kim and Aravas (1988) (Kim, K.S., Aravas, N., 1988, International Journal of Solids and Structures, 24, 417-435), a semianalytical expression for the work done by bending plasticity is proposed. The validity of the present expression is established based on finite element calculations. It is shown that for the interpretation of the results of peel test at 90 degrees when the peel force and the curvature are measured, the present approach can provide a precise value of the interface fracture energy.
\end{abstract}

*Corresponding author, e-mail: marion.martiny@univ-lorraine.fr 
Keywords : peel test, plastic dissipation, decohesion, Voce flow law, interface fracture energy

This is a post-peer-review, pre-copyedit version of an article published in International Journal of Fracture. The final authenticated version is available online at: https://doi.org/10.1007/s10704019-00393-7.

\section{Introduction}

The peel test is an affordable test conducted to determine the strength of the interface between two materials or between two layers. Depending on the mechanical behavior of the materials which form the interface, the prediction of the interface fracture energy from the force measurement is not always straightforward. A large effort has been provided in the literature to analyze in detail such experiments. For inextensible tape on a rigid substrate, Rivlin (1944) observed that the peel force during steady state provides a direct measure of the interface fracture energy. From an energy balance, Kendall (1973) and Kendall (1975) extended the Rivlin's approach to take into account the elastic contribution of the peel arm. Still in that configuration, the sole knowledge of the peel force provides an estimate of the interface fracture energy. The possibility of obtaining this interface property when complex material behaviors are present needs the evaluation of the work expenditure during peeling. An important contribution proposed by Kim and Aravas (1988) is the following. From the moment curvature relationship for the peel arm (assuming pure bending under plane strain), the work expenditure (or work done by bending plasticity) was obtained. They investigated the case of materials presenting hardening (powerlaw expression for the yield stress). Based on their work, the knowledge of the 
peel force and the slope of the film at the tip of the process zone is needed to predict the interface fracture energy. Kim et al. (1989) and Aravas et al. (1989) considered an elastic-perfectly plastic material. With this specific behavior, an explicit moment curvature relation during the bending and the reverse bending was obtained. In addition, a closed form expression for the work expenditure was proposed. As a consequence, from the energy balance of the peel test, the interface fracture energy was derived from the knowledge of two experimental observations: force and angle at the tip. This model has been adopted and discussed widely in the literature. Kinloch et al. (1994) analyzed the case where the film may also develop plasticity during the test. Instead of considering a power-law relation for the hardening behavior as in Kim and Aravas (1988), they considered that for a certain class of materials, the non-linear response of the material can be modeled by a bilinear relationship. Therefore, an explicit expression of the work done by bending plasticity was also found. The model was shown to be salient for a polyethylene film. Moidu et al. (1998) revisited this approach by still considering a film material having a bilinear elastic-plastic behavior. One may also cite Williams and Kauzlarich (2005) for considering pre-strain and also non-linear response. More recently, a large strain formalism has been proposed by Molinari and Ravichandran (2008) since during the decohesion process, the thin film may experience intense bending/reverse bending generating large strain.

Close to the crack tip, the assumption of pure bending response may be questionable. Therefore, Wei and Hutchinson (1998) adopted finite element calculations to analyze in detail the mechanical response of the system in the vicinity of the process zone. The detached arm was still modeled with the bending approach. The interface response was modeled by introducing cohesive elements. They also proposed a parametric analysis of the peel test based on 
non-dimensional parameters, see also Kim and Kim (1988). They showed that the interface fracture energy depends upon the material parameters (elastic and plastic responses) and also properties of the cohesive element. Thouless and Yang (2008) conducted also finite element simulations of the peel test, considering an elastic-plastic film. They evaluated precisely the relative importance of mode I and II components of the energy release rate in the cohesive zone. Wei (2004) compared the bending model of Kim and Aravas (1988) and Aravas et al. (1989) with the combined model of Wei and Hutchinson (1998). He extended the previous approach by taking into account the elastic compressibility. By still adopting a power-law relationship for the hardening response of the film, a semi-analytical expression was found for the link between moment and curvature during bending, but it was not possible to provide a simple expression for the reverse bending phase. So clearly, as the material description becomes more complex, the possibility to derive analytical or semi-analytical expression for the work done by bending plasticity is reduced. Wei and Zhao (2008) proposed a critical assessment of the different methods (bending versus combined bending + FE approaches). They illustrated their findings on specific configurations for metallic films made of aluminium or copper on a ceramic. Peel tests were conducted at various angles $\left(135^{\circ}\right.$ and $\left.180^{\circ}\right)$ in addition to 90 degrees. The thickness of the $\mathrm{Al}$ film was varying from 20 to 200 microns. They also considered ultra-thin film of copper deposited on a ceramic (from 1 to 15 microns). Based on previous works (Wei and Hutchinson (1998), Wei (2004)), they concluded that the combined model (cohesive element and bending of the free arm) is valid for a weak interface strength. For a strong interface case, a full finite element model is preferable. Zhao and Wei (2007) confirmed that a two-parameter model is needed to obtain the interface fracture energy from a peel test. Instead of measuring the base angle at the crack tip, they measured 
the curvature of the film during the bending/reverse bending process. With this information (force + curvature), the strength of the interface can be determined precisely. They were also able to propose values for the cohesive law parameters (interface fracture energy and separation strength). Experimental evidence based on copper thin films was discussed. To analyze T-peel test on $\mathrm{Cu} / \mathrm{Cr}$ / polyimide system, Song and $\mathrm{Yu}$ (2002) determined the mechanical response in tension of both $\mathrm{Cu} / \mathrm{Cr}$ and polyimide. The plastic response of $\mathrm{Cu}$ appeared to saturate at very small plastic strain so copper was modeled as an elastic-perfectly plastic material. From the theoretical expression of the work done by bending plasticity proposed by Kim and Aravas (1988), Aravas et al. (1989) and Kinloch et al. (1994), the interface fracture energy was obtained. For a similar configuration, one can also mention the work of Yang et al. (2000) on the symmetrical $90^{\circ}$-peel test on $\mathrm{Al}$ bonded by a thin adhesive layer.

From Wei and Hutchinson (1998) and Zhao and Wei (2007), when the material response is known, the most important parameters controlling the decohesion are the interface fracture energy and the strength of the interface. When the peel force and the curvature are measured in experiments, the interface fracture energy can be theoretically found. The goal of the paper is to propose a contribution to the precise definition of the work done by bending plasticity for a particular class of material responses. Indeed, as shown in Song and $\mathrm{Yu}$ (2002), the yield stress of copper saturates rapidly. As a consequence, instead of adopting an elastic-perfectly plastic response as proposed in Song and Yu (2002), we propose to adopt a Voce (1948) hardening law to mimic such saturation behavior. The paper is organized as follows. First the geometrical configuration and the material response are presented. In a second part, a semianalytical expression for the moment curvature during bending/reverse bending of metallic material, described by a Voce law, is proposed. Therefore, the work 
done by bending plasticity can be derived. Closed form expression is provided in some specific cases. Finally, the proposed expressions are compared to the Aravas et al. (1989) approach and validated based on finite element simulations of peel test.

\section{Configuration}

A two-layer woven composite/Cu sample is considered in this theoretical work. This configuration is representative of the one often present in printed circuit board. More generally, a system with a thick elastic substrate and a thin ductile film can also be investigated through the proposed approach. As shown in Fig. $1 \mathrm{a}$ ), the metallic film thickness $t$ is usually small compared to the one of the substrate ( $t$ between $17.5 \mu m$ and $70 \mu m$ ). Song and $\mathrm{Yu}(2002)$ characterized electroplated $\mathrm{Cu}$ layer under uniaxial tension. The measured uniaxial stress was saturating at early stage of the deformation. Based on this observation, they analyzed the T-peel test by adopting the elastic-perfectly plastic approach proposed by Aravas et al. (1989) and Kinloch et al. (1994). Instead of considering the material as an elastic-perfectly plastic one, we propose to model its plastic response by adopting a Voce (1948) law. In that case, the mechanical response is written as:

$$
\begin{aligned}
& \bar{\sigma}=E \bar{\epsilon} \quad \text { for } \quad \bar{\sigma} \leq \sigma_{o} \\
& \bar{\sigma}=\sigma_{o}+Q\left(1-\exp \left(-\gamma\left(\bar{\epsilon}-\epsilon_{o}\right)\right)\right) \text { for } \quad \bar{\sigma}>\sigma_{o}
\end{aligned}
$$

where $E$ is the Young modulus, $\bar{\sigma}$ the equivalent von Mises stress, $\bar{\epsilon}$ the accumulated strain and $\epsilon_{o}=\frac{\sigma_{o}}{E}$. The material is assumed incompressible. In the following of the paper, when plastic yielding is triggered, the yield stress (2) will 
be written as:

$$
\bar{\sigma}=\alpha-\beta \exp (-\gamma \bar{\epsilon})
$$

with $\alpha=\sigma_{o}+Q$ and $\beta=Q \exp \left(\gamma \epsilon_{o}\right)$. With the present modeling, the initial yield stress is $\sigma_{o}$ and the saturation value of the yield stress is $\alpha$, see Section 3 and Fig. 2 for an illustration of the adopted response. The present work can be viewed as an application of the bending approach proposed by Kim and Aravas (1988) for a specific material response presenting a saturation of the yield stress.

The moment curvature relationship during peeling test is analyzed assuming that 2D plane strain prevails, following the work of Kim and Aravas (1988), Aravas et al. (1989), Kinloch et al. (1994) or more recently Wei (2004). The main equations of the analysis are recalled below with emphasis of the novelty in the moment curvature relationship induced by considering the Voce law for plastic yielding. During the steady state phase of the test, any material point will experience a bending/reverse bending path, see Fig. 1 b). So the stress and strain tensors are (neglecting shear components):

$$
\boldsymbol{\sigma}=\left[\begin{array}{ccc}
\sigma_{11} & 0 & 0 \\
0 & \frac{\sigma_{11}}{2} & 0 \\
0 & 0 & 0
\end{array}\right] \quad \varepsilon=\left[\begin{array}{ccc}
\varepsilon_{11} & 0 & 0 \\
0 & 0 & 0 \\
0 & 0 & -\varepsilon_{11}
\end{array}\right]
$$

In the elastic domain, path (OA), from the Hooke's law and owing to incompressibility, one has $\sigma_{11}=\frac{4 E}{3} \epsilon_{11}$ while during plastic loading, $\left|\sigma_{11}\right|=\frac{2}{\sqrt{3}} \bar{\sigma}$. Pure bending is supposed to prevail. As in Kim and Aravas (1988), the axial strain is related to the position of the material within the cross-section by $\epsilon_{11}=-K z$ where $K$ is the curvature of the central surface (coordinate $z=0$ ) and $z$ is varying in the range $\left[-\frac{t}{2}, \frac{t}{2}\right]$. In Section 3 , it will be shown that for a certain range of material parameters, even if the pure bending approach is not fully justified, the work done by bending plasticity is still estimated accurately. 
This aspect was already mentioned by Kim and Kim (1988).

The bending moment per unit width of the film is classically given by:

$$
M=-\int_{-\frac{t}{2}}^{\frac{t}{2}} \sigma_{11} z d z=-2 \int_{0}^{\frac{t}{2}} \sigma_{11} z d z
$$

In the elastic phase of peeling (Path $O A$ in Fig. $1 \mathrm{~b}$ ), the moment curvature relationship is:

$$
M=\frac{E K t^{3}}{9} \quad 0 \leq K \leq K_{e}
$$

with the elastic limit curvature $K_{e}=\frac{\sqrt{3} \sigma_{o}}{E t}$.

For a ductile thin metallic film, the maximum curvature $K_{B}$ in point $\mathrm{B}$ of Fig. 1 b) is larger than $K_{e}$ so plasticity plays an important role and a precise calculation of the work done by bending plasticity is needed to extract the true (or objective as named in Kim and Kim (1988)) interface fracture energy $\Gamma$ from the measurement of the peel force. The moment curvature relationship during the plastic loading (path $A B$ ) is:

$$
M=-2 \int_{0}^{h} \sigma_{11} z d z-2 \int_{h}^{\frac{t}{2}} \sigma_{11} z d z
$$

where $h=\frac{\sigma_{o} \sqrt{3}}{2 E K}$ corresponds to the position of the elastic-plastic transition surface when the curvature $K$ is between $K_{e}$ and $K_{B}$. Note that during the plastic loading and due to $2 \mathrm{D}$ plane strain, the accumulated strain is $\bar{\epsilon}=\frac{2}{\sqrt{3}} K z$. Owing to the behavior (2) of the film, the expression (7) of the moment becomes:

$$
M=\frac{8 E K h^{3}}{9}+\frac{4}{\sqrt{3}} \int_{h}^{\frac{t}{2}}\left(\alpha-\beta \exp \left(-\frac{2 \gamma K z}{\sqrt{3}}\right)\right) z d z
$$

By integration by parts, the integral term on the right hand side of Eq. (8) is evaluated since

$$
\int_{z_{1}}^{z_{2}} z \exp (\delta z) d z=F\left(\delta, z_{2}\right)-F\left(\delta, z_{1}\right)
$$


with the function $F$ defined as $F(\delta, z)=\exp (\delta z)\left(\frac{z}{\delta}-\frac{1}{\delta^{2}}\right)$. As a consequence, an explicit expression for Eq. (8) is found:

$$
M=\frac{\alpha t^{2}}{2 \sqrt{3}}+\frac{\sigma_{o}^{2}}{E^{2} K^{2}}\left(\frac{\sigma_{o}}{\sqrt{3}}-\frac{\sqrt{3} \alpha}{2}\right)-\frac{4 \beta}{\sqrt{3}}\left[F\left(-\frac{2 \gamma K}{\sqrt{3}}, \frac{t}{2}\right)-F\left(-\frac{2 \gamma K}{\sqrt{3}}, h\right)\right]
$$

During the unloading phase, corresponding to path (BC) on Fig. 1 b) $\left(K_{B}-\right.$ $K_{B}^{*} \leq K \leq K_{B}$ ), the moment $M$ is linked to the curvature $K$ as in Kim and Kim (1988) by:

$$
M=M_{B}+\frac{E}{9}\left(K-K_{B}\right) t^{3}
$$

where $M_{B}=M\left(K=K_{B}\right)$ given in Eq. (10). In the following, we denote $h_{B}=$ $\frac{\sigma_{o} \sqrt{3}}{2 E K_{B}}$ the position of the elastic-plastic transition surface when the curvature is maximum and equal to $K_{B}$. The unloading phase ends in $\mathrm{C}$ when reverse plasticity develops at the outer surface of the film $z=\frac{t}{2}$. Let us denote $K_{C}=$ $K_{B}-K_{B}^{*} \leq K_{B}$ the curvature leading to this condition. Following Kim and Aravas (1988) or Kinloch et al. (1994), the curvature $K_{C}$ is defined as follows:

$$
\frac{2 \bar{\sigma}}{\sqrt{3}}=-\frac{2 \bar{\sigma}}{\sqrt{3}}+\frac{4 E}{3}\left(K_{B}-K_{C}\right) \frac{t}{2}
$$

where $\bar{\sigma}$ stands for the yield stress prevailing at $z=\frac{t}{2}$ at the end of plastic bending phase (i.e. for $\left.K=K_{B}\right): \bar{\sigma}=\alpha-\beta \exp \left(-\frac{\gamma K_{B} t}{\sqrt{3}}\right)$. As a consequence, one obtains: $K_{c}=K_{B}-K_{B}^{*}$ with $K_{B}^{*}=\frac{2 \sqrt{3}}{E t}\left(\alpha-\beta \exp \left(-\frac{\gamma K_{B} t}{\sqrt{3}}\right)\right)$

For the peel test, the reverse plastic loading, path (CD) on Fig. 1 b), exists since the free arm is pulled into straight plane with a final curvature of $K_{D}=0$. Let us denote $h^{\prime}(K)$ the position of the elastic-plastic transition surface during the reverse plastic loading. By construction, $h^{\prime}$ is larger than $h_{B}$, meaning that a material point with $z>h^{\prime}$ has already faced plasticity during the bending phase up to the curvature $K_{B}$. Similarly to Eq. (12), an implicit relationship 


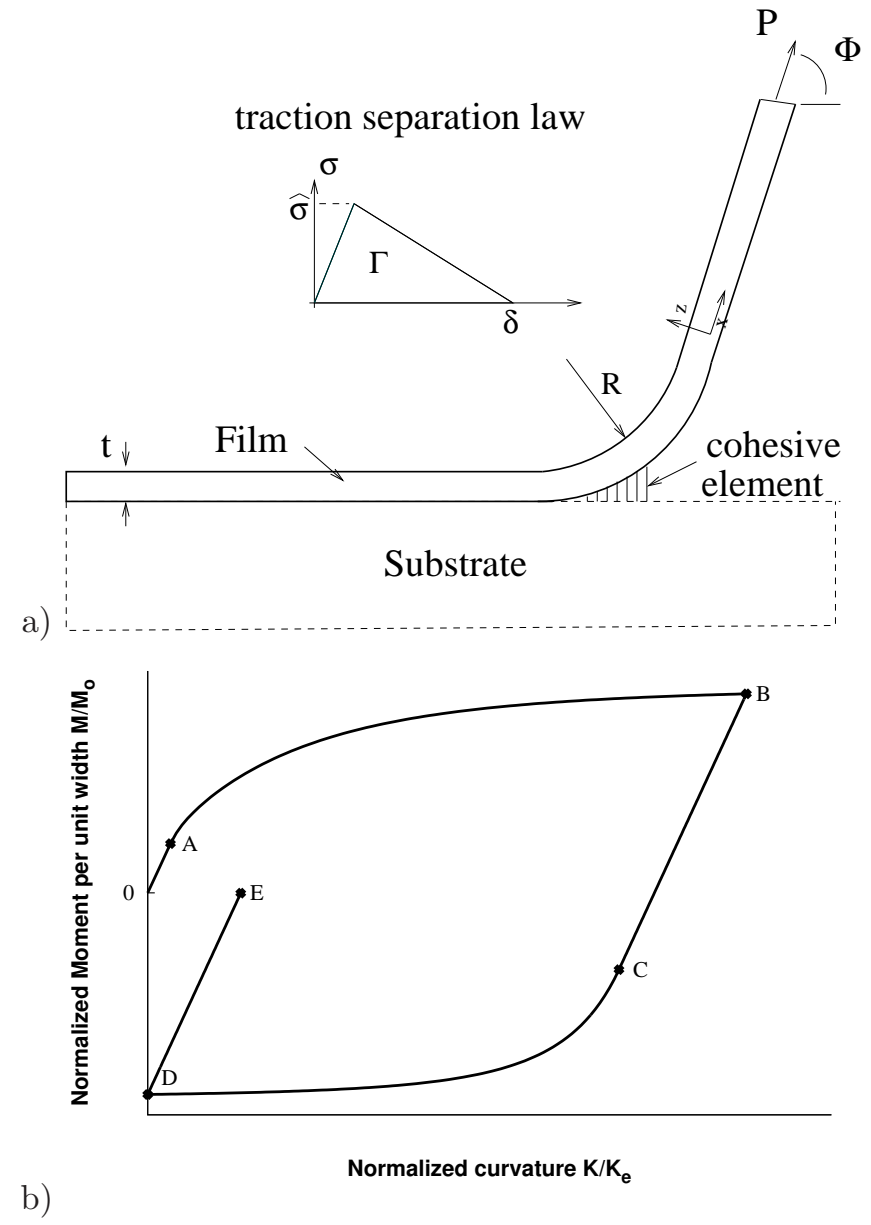

Figure 1: a) Peel test configuration. The film thickness is $t$. The peel angle is $\Phi$ and the force per unit width is $P$. The traction separation law is also presented where $\hat{\sigma}$ represents the peak stress and $\Gamma$ the interface fracture energy. A local coordinate system is adopted so that the position $z=0$ corresponds to the middle plane of the film. During peeling, the film is facing bending. Let us denote $R$ the radius of curvature of the top surface $z=\frac{t}{2}$. b) Schematic normalized moment versus normalized curvature under plane strain. Letters correspond to specific loading phases during the peel test.

for $h^{\prime}$ is found:

$$
\alpha-\beta \exp \left(-\frac{2 \gamma K_{B} h^{\prime}}{\sqrt{3}}\right)=\frac{E}{\sqrt{3}}\left(K_{B}-K\right) h^{\prime}
$$


In the theory as discussed by Aravas et al. (1989), the reverse plastic loading can continue for negative curvature. During peel test, according to Fig. 1 b), such a possibility is not considered. For a given curvature $K_{B}-K_{B}^{*} \geq K \geq 0$, the mechanical state of a material point in the film varies depending on its position. For $0 \leq z \leq h_{B}$, the material is elastic. For $h_{B} \leq z \leq h^{\prime}$, the material is also in the elastic regime but has faced some plasticity during the bending stage (AB). Finally, in the third part $h^{\prime} \leq z \leq \frac{t}{2}$ the material point develops reverse plasticity. For the latter part of the film, the accumulated strain is as given in Kim and Aravas (1988) and Kinloch et al. (1994):

$$
\bar{\epsilon}=\frac{2}{\sqrt{3}}\left[\left(2 K_{B}-K\right) z-\frac{\sqrt{3}}{E}\left(\alpha-\beta \exp \left(-\frac{2 \gamma K_{B} z}{\sqrt{3}}\right)\right)\right]
$$

By considering the three above strain histories, the moment $M$ can be evaluated in a semi-analytical manner:

$$
\begin{aligned}
M=\frac{8 E K_{B} h_{B}^{3}}{9} & -\frac{8 E}{9}\left(K_{B}-K\right)\left(h^{\prime}\right)^{3}+\frac{2 \alpha}{\sqrt{3}}\left(2\left(h^{\prime}\right)^{2}-h_{B}^{2}-\frac{t^{2}}{4}\right) \\
& -\frac{4 \beta}{\sqrt{3}}\left[F\left(-\frac{2 \gamma K_{B}}{\sqrt{3}}, h^{\prime}\right)-F\left(-\frac{2 \gamma K_{B}}{\sqrt{3}}, h_{B}\right)\right]+\frac{4 \beta}{\sqrt{3}} \int_{h^{\prime}}^{\frac{t}{2}} z \exp (-\gamma \bar{\epsilon}) d z
\end{aligned}
$$

with $h^{\prime}$ defined in Eq. (13) and $\bar{\epsilon}$ in Eq. (14). Note that when the curvature $K_{B}$ and the parameter $\gamma$ are large and the ratio $\frac{\sigma_{o}+Q}{E}$ is small (situation which is observed for peel test of copper film on an elastic substrate), the accumulated strain can be approximated by:

$$
\bar{\epsilon}=\frac{2}{\sqrt{3}}\left(2 K_{B}-K\right) z
$$

In that case, a closed form expression for $M$ defined in Eq. (15) is available. Indeed the integral term of the right hand side of Eq. (15) becomes:

$$
\int_{h^{\prime}}^{\frac{t}{2}} z \exp (-\gamma \bar{\epsilon}) d z=F\left(-\frac{2 \gamma\left(2 K_{B}-K\right)}{\sqrt{3}}, \frac{t}{2}\right)-F\left(-\frac{2 \gamma\left(2 K_{B}-K\right)}{\sqrt{3}}, h^{\prime}\right)
$$


The domain of validity of this approximate solution will be discussed in Section 3.

Eqs (6), (10), (11) and (15) provide the moment curvature expressions for a Voce hardening law. Two limiting cases have been explored in the literature because of the possibility to derive analytical expressions for the moment curvature relation and therefore for the work done by plastic bending. First, when the material is elastic-perfectly plastic with $Q=0$ in Eq. (2) (i.e. $\alpha=\sigma_{o}$ and $\beta=0$ ), the corresponding relationships provided by Aravas et al. (1989) and Kim and Kim (1988) are recovered. Second, when the parameter $\gamma$ is small, the Voce hardening law can be replaced by its first order Taylor expansion:

$$
\bar{\sigma}=\sigma_{o}+Q \gamma\left(\bar{\epsilon}-\bar{\epsilon}_{o}\right)
$$

After some calculations, it can be shown that the moment curvature expressions derived by Kinloch et al. (1994) for bilinear material are also obtained.

As proposed by Kendall (1973), Aravas et al. (1989) or Wei and Hutchinson (1998) among others of the literature, the energy balance provides a link between the peel force per unit width $P$ (see Fig. 1 a), the interface fracture energy $\Gamma$ and the work done by bending plasticity $\Psi$ :

$$
P(1-\cos \Phi)=\Gamma+\Psi \quad \Rightarrow \quad \Gamma=P(1-\cos \Phi)-\Psi
$$

Eq. (19) is obtained assuming that the change of elastic strain energy due to the extension of the film under the peel force $P$ is negligible. The term $\Psi$ contains the plastic dissipation and the residual strain energy contribution during plastic bending, see Kim and Aravas (1988), Kim et al. (1989) or Martiny et al. (2008). As mentioned by Kim and Aravas (1988), the work done by bending plasticity (or the work expenditure) per unit crack advance is $\Psi=\int_{L} M d K$ where $L$ is 
the loading path $(\mathrm{OABCD})$ in the moment curvature of Fig. $1 \mathrm{~b}$ ):

$$
\Psi=\int_{o}^{K_{e}} M d K+\int_{K_{e}}^{K_{B}} M d K+\int_{K_{B}}^{K_{B}-K_{B}^{*}} M d K+\int_{K_{B}-K_{B}^{*}}^{0} M d K
$$

Interestingly, the moment curvature relationship is known for the whole path $(\mathrm{OABCD})$. Owing to the explicit expression on the path $(\mathrm{OABC})$, the first three terms on the right hand side of Eq. (20) can be evaluated analytically. More details can be found in Appendix A. Finally, we obtained:

$$
\begin{aligned}
& \Psi=\frac{\sigma_{o}^{2} t}{6 E}+ \frac{\alpha t^{2}}{2 \sqrt{3}}\left(K_{B}-K_{e}\right)+\left(\frac{\sigma_{o}}{\sqrt{3}}-\frac{\sqrt{3} \alpha}{2}\right) \frac{\sigma_{o}^{2}}{E^{2}}\left(\frac{1}{K_{e}}-\frac{1}{K_{B}}\right) \\
&- \frac{\sqrt{3} \beta}{\gamma^{2}}\left[G\left(-\frac{\gamma t}{\sqrt{3}}, K_{B}\right)-G\left(-\frac{\gamma t}{\sqrt{3}}, K_{e}\right)\right]+\frac{\sqrt{3} \beta}{\gamma} \exp \left(-\frac{\gamma \sigma_{o}}{E}\right) \\
&\left(\frac{\sigma_{o}}{E}+\frac{1}{\gamma}\right)\left(\frac{1}{K_{B}}-\frac{1}{K_{e}}\right)-M_{B} K_{B}^{*}+\frac{E t^{3}}{18}\left(K_{B}^{*}\right)^{2}+\left[\frac{8 E K_{B} h_{B}^{3}}{9}\right. \\
&\left.-\frac{2 \alpha}{\sqrt{3}}\left(h_{B}^{2}+\frac{t^{2}}{4}\right)+\frac{4 \beta}{\sqrt{3}} F\left(-\frac{2 \gamma K_{B}}{\sqrt{3}}, h_{B}\right)\right]\left(K_{B}^{*}-K_{B}\right)+I
\end{aligned}
$$

where the function $G(x, y)$ is defined as $G(x, y)=\frac{\exp (x y)}{y}$ and $I$ given by:

$$
\begin{gathered}
I=\int_{K_{B}-K_{B}^{*}}^{0}\left[\frac{8 E}{9}\left(K-K_{B}\right)\left(h^{\prime}\right)^{3}+\frac{4 \alpha}{\sqrt{3}}\left(h^{\prime}\right)^{2}\right. \\
\left.-\frac{4 \beta}{\sqrt{3}} F\left(-\frac{2 \gamma K_{B}}{\sqrt{3}}, h^{\prime}\right)+\frac{4 \beta}{\sqrt{3}} \int_{h^{\prime}}^{\frac{t}{2}} z \exp (-\gamma \bar{\epsilon}) d z\right] d K
\end{gathered}
$$

The expression (21) is semi-analytical. Based on the present derivation, the work done by bending plasticity $\Psi$ has almost a closed form expression except for the last term. Indeed, $h^{\prime}$ defined by the implicit relationship (13) is a function of the current curvature $K$. Nevertheless, when the radius of curvature is small ( $K_{B}$ large) and the parameter $\gamma$ has an important value, the expression for the accumulated strain (14) is given by Eq. (16). In addition we propose to define $h^{\prime}$ of Eq. (13) by $h^{\prime}=\frac{\sqrt{3} \alpha}{E\left(K_{B}-K\right)}$. Based on these two assumptions, the last 
integral term $I$ can be evaluated in closed form:

$$
\begin{array}{r}
I^{\text {approx }}=\frac{4 \alpha^{3}}{\sqrt{3} E^{2}}\left(\frac{1}{K_{B}}-\frac{1}{K_{B}^{*}}\right)-\frac{\sqrt{3} \beta}{\gamma^{2}}\left(G\left(-\frac{\gamma t}{\sqrt{3}}, 2 K_{B}\right)\right. \\
\left.-G\left(-\frac{\gamma t}{\sqrt{3}}, K_{B}+K_{B}^{*}\right)\right)+\frac{\sqrt{3} \beta}{K_{B} \gamma^{2}}\left(G\left(-2 \frac{\gamma \alpha}{E}, \frac{K_{B}}{K_{B}^{*}}\right)\right. \\
\left.-G\left(-2 \frac{\gamma \alpha}{E}, 1\right)+G\left(-2 \frac{\gamma \alpha}{E}, \frac{K_{B}+K_{B}^{*}}{K_{B}^{*}}\right)-G\left(-2 \frac{\gamma \alpha}{E}, 2\right)\right)
\end{array}
$$

The present approach also contains, by construction, the elastic-perfectly plastic case. Indeed, when the parameter $Q$ of Eq. (2) is set to zero, the material is obviously elastic-perfectly plastic. In that case, since $\alpha=\sigma_{o}$, one obtains $K_{B}^{*}=2 K_{e}, h_{B}=\frac{\sigma_{o} \sqrt{3}}{2 E K_{B}}$. It is easily shown that the expression of $\Psi$ is identical to the expression provided by Aravas et al. (1989) or Kim and Kim (1988) for an elastic-perfectly plastic material:

$$
\Psi^{P P}=M_{o} K_{e}\left(2 \frac{K_{B}}{K_{e}}-5+\frac{10}{3} \frac{K_{e}}{K_{B}}\right) \quad \text { when } \quad K_{B} \geq 2 K_{e}
$$

where $M_{o}=\frac{1}{2 \sqrt{3}} \sigma_{o} t^{2}$ is the fully plastic moment. As discussed previously, the present approach also contains the bilinear response for an elastic-plastic material proposed by Kinloch et al. (1994) or Moidu et al. (1998) when the parameter $\gamma$ is small.

When $\gamma$ is large, the saturation of the yield stress occurs at small strain. Therefore, it can be argued that the material is almost elastic-perfectly plastic with a yield stress being equal to the saturation stress $Q+\sigma_{o}$. This assumption was adopted by Song and Yu (2002). This case will be discussed in Section 3. One can also mention that the extension of the present approach to a constitutive behavior containing a finite number of Voce law:

$$
\bar{\sigma}=\sigma_{o}+\sum_{i=1}^{N} Q_{i}\left(1-\exp \left(-\gamma_{i}\left(\bar{\epsilon}-\epsilon_{o}\right)\right)\right)
$$


is straightforward. This can be done by imposing $\alpha=\sigma_{o}+\sum_{i=1}^{N} Q_{i}$, and by replacing $\beta$ by $\beta_{i}=Q_{i} \exp \left(\gamma_{i} \epsilon_{o}\right)$ and $\gamma$ by $\gamma_{i}$. For the sake of brevity, corresponding expressions with the summation are not reproduced here.

\section{Results}

The first part of this section is devoted to the evaluation of the moment curvature response of a thin film under bending/reverse bending. The difference between the present development based on a Voce yield stress with the one of Kim and Kim (1988) for elastic-perfectly plastic response is illustrated. In a second part, the interface fracture energy estimated based on the work done by bending plasticity is compared to the one imposed in finite element simulations of the peel test at angle $\Phi=90^{\circ}$. Finally, the effect of the parameters of cohesive law on the peel force and curvature is investigated for peel tests at various angles $\Phi$ in the range $\left[45^{\circ} ; 135^{\circ}\right]$.

\subsection{Predictions of the model}

The effect of the parameter $\gamma$ of Eq. (2) on the elastic-plastic response of the metallic film is first investigated in Fig. 2. The reference parameters for the elastic-plastic behavior of the film are listed in Table 1. The parameter $\gamma$ influences the deformation level for which the yield stress approaches the plateau $\sigma_{o}+Q$. Indeed for a small value of $\gamma($ here $\gamma=5$ ), a large strain is needed to reach the saturation regime (around $\bar{\epsilon}=1$ ), while for a large value of $\gamma$ (here $\gamma=160$ ), a small strain is sufficient for the yield stress to reach the plateau (around $\bar{\epsilon}=0.03)$. The initial plastic hardening modulus $\frac{\partial \bar{\sigma}}{\partial \bar{\epsilon}}\left(\bar{\epsilon}=\epsilon_{o}\right)$ is highly affected by the value of $\gamma$. For a metal, it must be lower than the Young's modulus, leading to the condition for $\gamma: \gamma \leq \frac{E}{Q}$. With the present material parameters of Table 1, one obtains: $\gamma_{\text {lim }}=361$. 
Table 1: Reference parameters for the elastic-plastic behavior of the metallic film and for the cohesive law

\begin{tabular}{|c|c|c|c|c|c|c|c|}
\hline$t$ & $E$ & $\nu$ & $\sigma_{o}$ & $Q$ & $\gamma$ & $\Gamma$ & $\hat{\sigma}$ \\
$\mu m$ & $G P a$ & & $M P a$ & $M P a$ & & $N / m$ & $M P a$ \\
\hline 35 & 65 & 0.26 & 100 & 180 & $5-160$ & 1055 & 10 \\
\hline
\end{tabular}

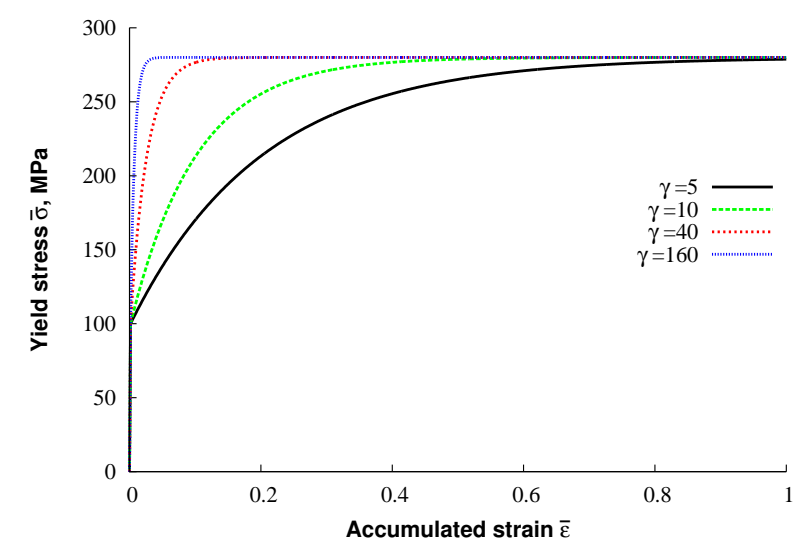

Figure 2: Elastic-plastic behavior of the metallic film. A Voce hardening law Eq. (2) is considered. Depending on the value of $\gamma$, the saturation state is reached for different accumulated strains.

It is also interesting to observe the difference between the moment curvature evolution predicted by adopting a Voce hardening law and by assuming that the material is elastic-perfectly plastic. For illustration, in Fig. 3, the curvature of the metallic film is $K_{B}=4000 \mathrm{~m}^{-1}$, leading to a radius of $0.25 \mathrm{~mm}$. The film thickness is $t=35 \mu \mathrm{m}$. Two elastic-perfectly plastic cases are considered. The first one assumes that the yield stress of the material is $\bar{\sigma}=\sigma_{o}$ while in the second case it becomes $\bar{\sigma}=\sigma_{o}+Q$. As expected, the response based on the Voce law is located between the two curves corresponding to elasticperfectly plastic materials. Since the work done by bending plasticity $\Psi$ is the area formed by the curve along the path (OABCD), it is clear that assuming 
an elastic-perfectly plastic behavior for the material is only salient for very large values of $\gamma$. It is interesting to notice that due to strain hardening, the moment of curvature response during the reverse bending (path CD of Fig. 1 b) for $\gamma=160$ corresponds closely to the response of an elastic-perfectly plastic material with $\bar{\sigma}=\sigma_{o}+Q$. Nevertheless, during bending (path $\mathrm{AB}$ of Fig. 1 b), some differences exist which affect the work $\Psi$. On the contrary, for $\gamma=40$, the work done by bending plasticity $\Psi$ is strongly overestimated by assuming an elastic-perfectly plastic response with $\bar{\sigma}=\sigma_{o}+Q$. Fig. 3 b) displays the evolution of $\Psi$ as $\gamma$ is enlarged, keeping $K_{B}=4000 \mathrm{~m}^{-1}$. It is observed that $\Psi$ is increasing rapidly for small values of $\gamma$. Indeed, as $\gamma$ becomes larger, the response of the material becomes closer to an elastic-perfectly plastic response, see Fig. 2. As a consequence, the value of $\Psi$ saturates, reaching $\Psi^{P P}$ predicted by the elastic-perfectly plastic approach of Aravas et al. (1989) and Kim and Kim (1988). These trends are confirmed for two values of $Q: Q=90$ or $180 M P a$.

For material parameters of Table 1 and two values of $\gamma: 40$ and 160, the work done by bending plasticity evaluated based on Eqs. (21) and (22) is presented in Fig. 4 a) for a curvature $K$ in the range $1000 \mathrm{~m}^{-1}$ to $10000 \mathrm{~m}^{-1}$ leading to a radius of the film varying from $100 \mu \mathrm{m}$ to $1 \mathrm{~mm}$. As expected, $\Psi$ is larger when the normalized curvature is increased. For large value of the normalized curvature, the response is seen to be almost linear for both values of $\gamma$. This could have been anticipated. Indeed, Fig. 3 a) has shown that for large $\gamma$, the moment versus curvature response is quite similar to the one estimated based on an elastic-perfectly plastic approach. Therefore, in that case, the work $\Psi$ for a Voce law or for an elastic-perfectly plastic material (with $\bar{\sigma}=\sigma_{o}+Q$ ) must be quite similar. From Eq. (24), the increment of work $\Delta \Psi$ produced by an increment of the normalized curvature $\frac{\Delta K}{K_{e}}$ is expressed as follows, when $\frac{K}{K_{e}}$ 


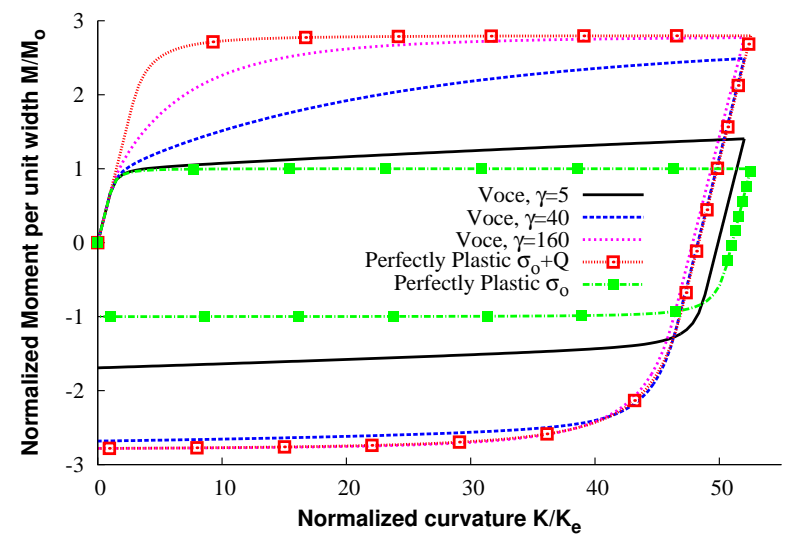

a)

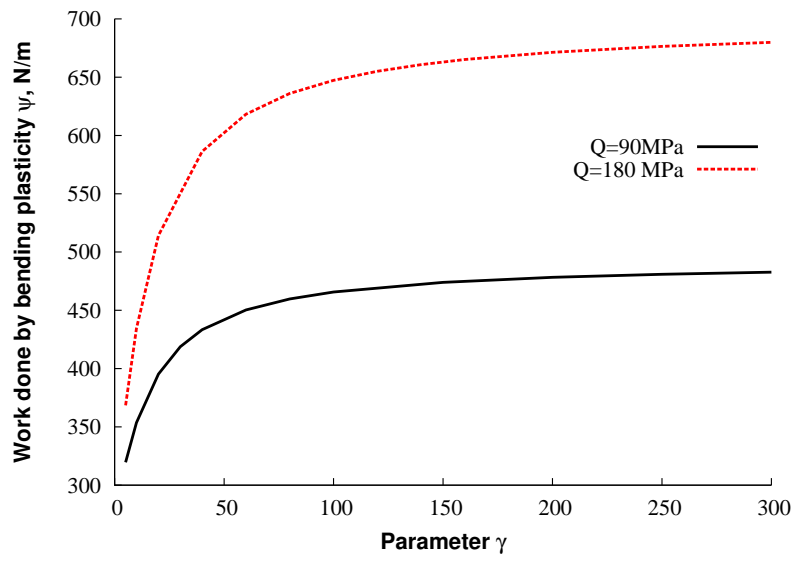

Figure 3: a) Estimated moment curvature relationship for an elastic-perfectly plastic material and a material with a Voce yield stress. Effect of the parameter $\gamma$ controlling the deformation for which the yield stress saturates, see Eq. (2). b) Work done by bending plasticity as a function of the parameter $\gamma$ for two values of $Q=90 M P a$ and $Q=180 M P a . M_{o}$ is the fully plastic moment. $K_{e}$ is the elastic limit curvature.

is large:

$$
\Delta \Psi=2 M_{o} \Delta K
$$

With the parameters of Table 1 , we observe that $\Delta \Psi \approx 15 \frac{\Delta K}{K_{e}}$. This is consistent with the observation of Fig. 4 a). In addition, for the two values $\gamma=40$ and $\gamma=160$, we have found that the approximate relationship for $\Psi$ based on Eq. 
(23) almost coincides with the one obtained with Eq. (21) (results not presented here).

Next, a more precise comparison between the predicted work done by bending plasticity $\Psi$ evaluated using the Voce law or an elastic-perfectly plastic response $\left(\psi^{P P}\right.$ with $\left.\bar{\sigma}=\sigma_{o}+Q\right)$ is presented in Fig. 4 b). For the value $\gamma=40$, the normalized ratio $\frac{\psi}{\psi^{P P}}$ is below 0.9 when the normalized curvature $\frac{K}{K_{e}}$ is equal to $88\left(K=6676 m^{-1}\right.$ or a radius of $\left.149 \mu m\right)$. Adopting an elasticperfectly plastic aproximation for this material when analyzing results of the peel test will lead to a $10 \%$ difference for the interface fracture energy $\Gamma$, see the energy balance equation (19) for a test at $\Phi=90^{\circ}$. When $\gamma=160$, we clearly observe that for $\frac{K}{K_{e}}=88$, less than $3 \%$ difference remains. Note that Fig. 3 is built with a value of the curvature of $K_{B}=4000 m^{-1}\left(\frac{K_{B}}{K_{e}}=52\right)$. In that case, a $15 \%$ difference exists between $\Psi$ based on expression (21) and $\Psi^{P P}$ for $\gamma=40$ while for $\gamma=160$, a $4 \%$ difference remains.

\subsection{Finite element validation based on peel test}

In this section, we analyze the peel test for a two-layer sample made of woven composite and copper. The plastic behavior of copper is described by Eq. (2). The substrate, representative of a woven composite used in the printed circuit board industry, remains elastic. Its elastic behavior has been identified based on the strategy proposed by Girard et al. (2018). The in-plane Young's modulus is around 20.6 GPa, while the out-of-plane one is $16.4 \mathrm{GPa}$. The in-plane Poisson's ratio is 0.17 .

A Finite Element (FE) model of the peel test under plane strain conditions has been considered on ABAQUS Software for various angles $\Phi$, see Fig. 1 a). The interface is modeled with cohesive elements: a bilinear traction-separation 


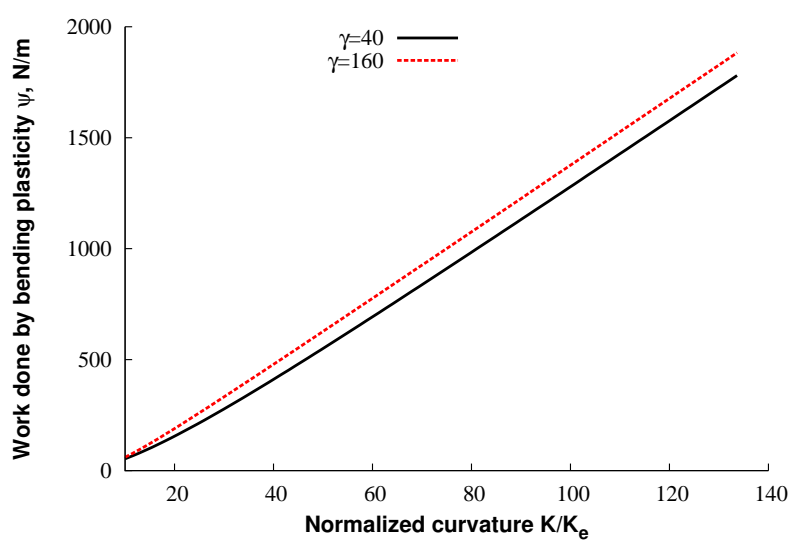

a)

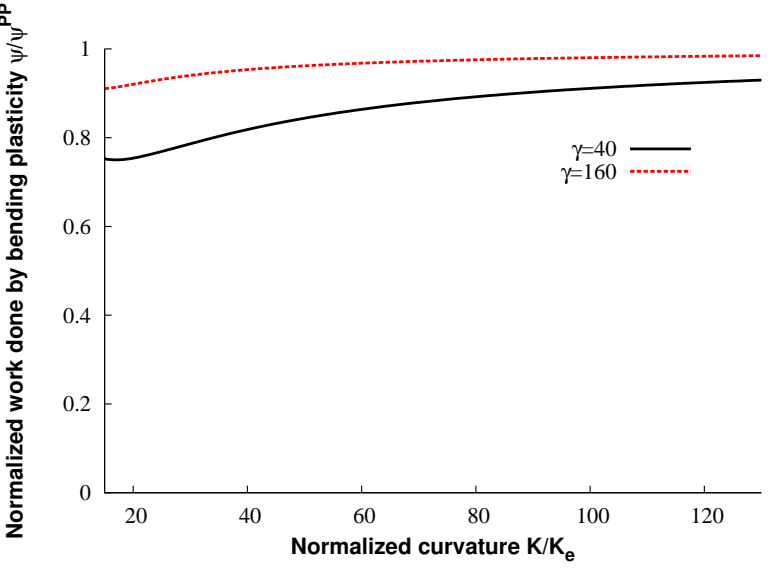

b)

Figure 4: a) Work done by bending plasticity $\Psi$ versus normalized curvature $K / K_{e}$ for two values of $\gamma: \gamma=40$ and $\gamma=160$. b) Ratio between the work done by bending plasticity evaluated based on a Voce law and an elastic-perfectly plastic response. Material parameters for the Voce law are given in Table 1.

law in mixed mode is considered. Before failure initiation, the traction separation law adopted here is such that $\sigma_{n}=K_{n n} \delta_{n}$ and $\sigma_{t}=K_{t t} \delta_{t}$. In the present work, we adopt $K_{t t}=K_{n n}=10^{6} \mathrm{~N} / \mathrm{mm}^{3} . \sigma_{n}, \sigma_{t}$ are respectively the normal and tangential components of the traction force. $\delta_{n}$ and $\delta_{t}$ are the normal and sliding displacements at the level of the cohesive element. The following 
quadratic failure initiation and linear failure criteria are adopted :

$$
\left(\frac{\sigma_{n}}{\hat{\sigma}_{n, c}}\right)^{2}+\left(\frac{\sigma_{t}}{\hat{\sigma}_{t, c}}\right)^{2}=1 \quad \frac{G_{n}}{\Gamma_{I}}+\frac{G_{t}}{\Gamma_{I I}}=1
$$

with $G_{n}=\int \sigma_{n} d \delta_{n}$ and $G_{t}=\int \sigma_{t} d \delta_{t} . \Gamma_{I}$ (resp. $\Gamma_{I I}$ ) is the mode I toughness (resp. mode II toughness). The adopted cohesive law is implemented in ABAQUS (2013) software. More information can be found elsewhere. Note that other choices for the cohesive law could have been adopted, see Ortiz and Pandolfi (1999) or Thouless and Yang (2008). Discussion about mixed mode ratio can also be found in Tvergaard and Hutchinson (1993), Kinloch et al. (1994), Yang and Thouless (2001), Wei (2004) and Martiny et al. (2008). In our FE simulations, as in most of the literature on the subject, only two parameters are kept for the definition of the cohesive law by imposing: $\hat{\sigma}_{n, c}=\hat{\sigma}_{t, c}=\hat{\sigma}$ and $\Gamma_{I}=\Gamma_{I I}=\Gamma . \hat{\sigma}$ represents the peak stress and $\Gamma$ the interface fracture energy, see Fig. 1 a).

The goal of the finite element simulations is to provide values of the peel force and the curvature for a given cohesive law and a given material behavior for the thin film. Knowing the curvature, the work done by bending plasticity $\Psi$ can be evaluated by Eq. (21). Subsequently, the interface fracture energy $\Gamma$ is derived from the energy balance (19). The predicted value for $\Gamma$ is compared to the one adopted in FE simulations. The reference parameters for the cohesive law and for the elastic-plastic behavior of the film are listed in Table 1.

Four-node plane strain elements (CPE4R) are adopted with 9 elements in the film thickness for $\Phi<90^{\circ}$. For $\Phi \geq 90^{\circ}$, 18 elements in the film thickness are considered since the curvature is smaller (sometimes around $100 \mu m$ for $\Phi=$ $135^{\circ}$ ). A mesh sensitivity study has been carried out by considering larger mesh density. By increasing the number of elements, and therefore by reducing the 
element size, the predictions in terms of peel force and curvature are preserved. The cohesive element size in the 1 direction is five times lower than the element size in the film. The height of the cohesive elements is vanishingly small. In all calculations, the velocity for the peel arm is $V=0.5 \mathrm{~mm} / \mathrm{s}$. To ensure that the peel angle $\Phi$ remains constant during the FE simulation, the substrate is moving in the horizontal direction with the same velocity $V=0.5 \mathrm{~mm} / \mathrm{s}$. Note that the length of the substrate is $50 \mathrm{~mm}$ long. It has been checked that a stationary process prevails after quite a short period of time.

First, we present a comparison of the model capability based on a peel test with $\Phi=90^{\circ}$. This working condition is most often used in the literature and for practical applications. For instance, a peel test at $\Phi=90^{\circ}$ is recommended by the Institute for Interconnecting and Packaging Electronic Circuits via the IPC-TM-650 2.4.8 standard on measuring the peel strength of metallic clad laminates, IPC (1994). The force versus time is presented in Fig. 5 for three values of $\gamma$ : 5, 40 and 160 and for two values of $Q$ : 90 and $180 \mathrm{MPa}$. A stationary process for the peel force is established after $3 \mathrm{~s}$. We clearly observe that for the same interface fracture energy $\Gamma=1055 \mathrm{~N} / \mathrm{m}\left(\right.$ or $\left.J / \mathrm{m}^{2}\right)$, the force is larger when $\gamma$ and/or $Q$ increase. Indeed, for $Q=180 M P a$, the level of the force evolves from $1531 \mathrm{~N} / \mathrm{m}$ with $\gamma=5$ to $1707 \mathrm{~N} / \mathrm{m}$ for $\gamma=160$. From the energy balance (19), and since $\Gamma$ is fixed, this enhancement of the force means that the work done by bending plasticity $\Psi$ is larger.

A key factor controlling $\Psi$ is the maximum curvature $K_{B}$, existing at the end of the loading path $(\mathrm{AB})$. During the stationary process in FE simulations, the radius of curvature $R$ of the top surface $z=\frac{t}{2}$ can be measured. The area where the thin film is detached from the substrate is presented in Fig. 5 b). It is observed in this figure, that in the case of a peel test with an angle of $\Phi=90^{\circ}$, the film is curved with a radius $R$, which is assumed to be representative of 


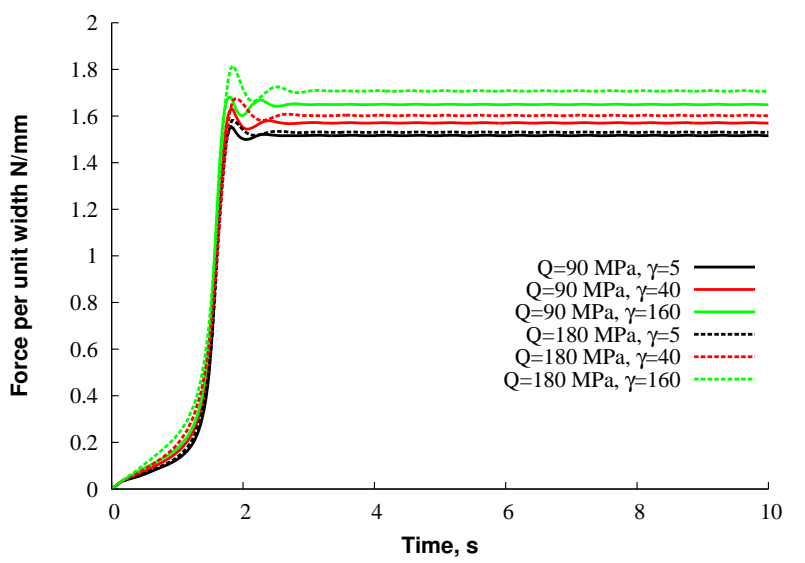

a)

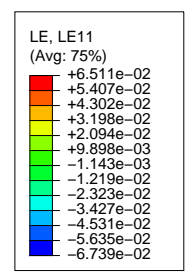

b)

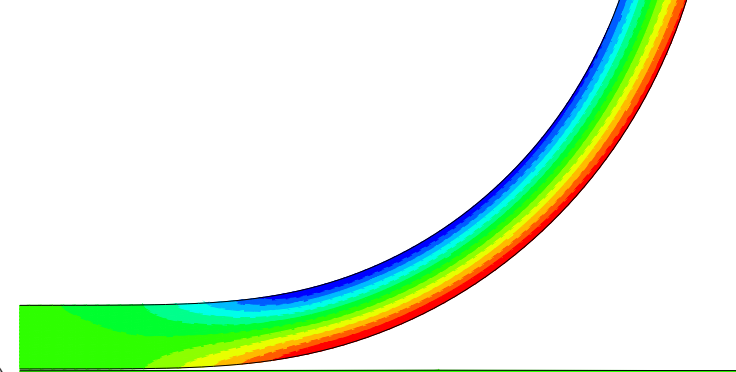

Figure 5: a) Force per unit width versus time. Different values of material parameters $Q$ and $\gamma$ are tested. b) View of the film curvature during the bending/reverse bending process. The cohesive elements are removed for presentation purposes. The axial strain $\epsilon_{11}$ is displayed. A local orientation is adopted so that the direction 1 is always parallel to the central surface, as presented in Fig. 1 .

the curvature at point $B$ of Fig. 1 b). To be consistent with the bending model where the curvature is measured from the central surface of the film, the curvature $K_{B}$ is linked to $R$ by $K_{B}=\left(R+\frac{t}{2}\right)^{-1}$. Fig. 6 presents the corresponding evolution of the curvature versus $\gamma$ for the two different values 
of $Q$. The evolution of the curvature versus $\gamma$ is not monotonic. For moderate values of $\gamma, K_{B}$ is first decreasing. As $\gamma$ becomes larger, $K_{B}$ reaches a minimum and subsequently increases slightly. Note also that depending on the respective value of $Q$ which is scaling the saturation of the flow stress, the minimum of the curve is reached for larger value of $\gamma$ as $Q$ increases. The non-monotonic trend can be understood as follows. On the one hand, from Fig. 5, it is seen that when $\gamma$ is increased from 5 to 40 , the level of the force is slightly increased. From the balance of energy, a larger force level is linked to an increase in work done by bending plasticity $\Psi$. Nevertheless, in Fig. 3 b), it has been shown that, when $\gamma$ is increased from 5 to $40, \Psi$ is strongly enhanced for a fixed curvature. As a consequence, to preserve the balance of energy and the slight increase of the force, the curvature is reduced in order to propose a moderate increase in $\Psi$. On the other hand, when $\gamma$ is large, the value of $\Psi$ saturates, assuming that the curvature remains fixed, see Fig. $3 \mathrm{~b}$ ). Therefore, the larger peel force observed for $\gamma=160$ when compared to $\gamma=40$ originates from the increase of the curvature, which induces larger work done by bending plasticity.

The prediction of the work done by bending plasticity can be obtained via Eq. (21) based on the curvature $K_{B}$ captured by the finite element calculation (Fig. 6). In addition, from the numerical measurement of the peel force (Fig. 5), and from Eq. (19), the interface fracture energy $\Gamma$ is estimated. The result of the dialog between the present model and the finite element calculation is illustrated in Table 2. We collect in this table three predictions for $\Gamma$ based, first on the estimate of $\Psi$ provided by the current approach Eq. (21), second, on Eq. (23) and a third one, based on the elastic-perfectly plastic assumption for the film behavior, Eq. (24). The obtained values are compared to $\Gamma=1055 \mathrm{~N} / \mathrm{m}$, which is the prescribed value in the finite element simulations. All the results 


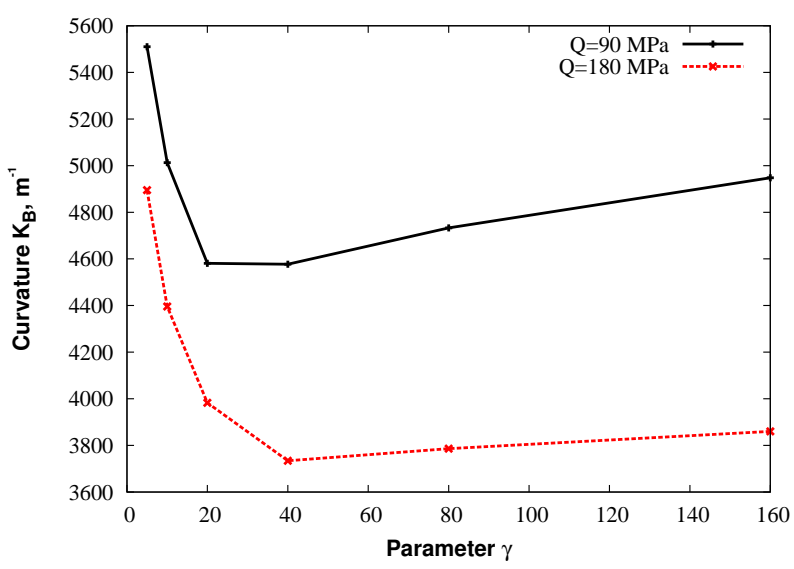

Figure 6: Effect of the $\gamma$ parameter of Eq. (2) on the curvature $K_{B}$ of the film during a peel test at $\Phi=90^{\circ}$. The ductile film behavior is elastic-plastic with a yield stress described by a Voce law (2). Material parameters are listed in Table 1.

are provided based on a $90^{\circ}$ peel test. Our present approach is able to predict the $\Gamma$ value with less than $1.5 \%$ in the whole range of $\gamma$ for the two considered cases $Q=90 M P a$ and $Q=180 M P a$, see Table 2. As expected, adopting the formula (24) proposed by Aravas et al. (1989) is only valid when $\gamma$ is larger than 40. In that case, the stress-strain response in tension is quite similar to the one expected when the material has an elastic-perfectly plastic behavior, see Fig. 2. Note also that the estimate of the interface fracture energy based on the approximate solution Eq. (23) is also accurate for the values of the $\gamma$ parameter considered in the present paper. This observation confirms a previous finding where a limited difference has been observed between predictions of the interface fracture energy based on Eqs (21) and (23), see results of Fig. 4. Fig. 7 a) presents the time evolution of the axial strain component $\epsilon_{11}$ for material points located on the upper part $(z=16.6,14.6$ and $12.6 \mu m)$ and on the lower part $(z=-16.6,-14.6$ and $-12.6 \mu m)$ of the film cross-section. As expected, material points located at the lower (resp. upper) part of the film 
Table 2: Predicted interface fracture energy. From the force and curvature measured owing to finite element simulations (here $\Gamma=1055 \mathrm{~N} / \mathrm{m}, \Phi=90^{\circ}$ ), the work $\Psi$ is evaluated based on the present approach (21), on the approximate solution (23) and on the elastic-perfectly plastic approach of Aravas et al. (1989), see Eq. (24). From the energy conservation (19), the interface fracture energy is estimated.

Estimated $\Gamma(\mathrm{N} / \mathrm{m})$ for $Q=90 M P a$

\begin{tabular}{|ll|c|c|c|c|c|c|}
\hline$\gamma$ & & 5 & 10 & 20 & 40 & 80 & 160 \\
\hline $\begin{array}{l}\Gamma \quad \text { from } \\
(21)\end{array}$ & Eq. & 1056 & 1053 & 1050 & 1054 & 1046 & 1044 \\
\hline $\begin{array}{l}\Gamma \quad \text { from } \\
(23)\end{array}$ & Eq. & 1051 & 1050 & 1048 & 1053 & 1045 & 1043 \\
\hline$\Gamma^{P P}(24)$ & & 831 & 891 & 946 & 995 & 1014 & 1028 \\
\hline
\end{tabular}

Estimated $\Gamma(\mathrm{N} / \mathrm{m})$ for $Q=180 M P a$

\begin{tabular}{|ll|c|c|c|c|c|c|}
\hline$\gamma$ & & 5 & 10 & 20 & 40 & 80 & 160 \\
\hline $\begin{array}{l}\Gamma \text { from } \\
(21)\end{array}$ & Eq. & 1052 & 1047 & 1050 & 1058 & 1069 & 1066 \\
\hline $\begin{array}{l}\Gamma \quad \text { from } \\
(23)\end{array}$ & Eq. & 1031 & 1035 & 1044 & 1055 & 1060 & 1066 \\
\hline$\Gamma^{P P}(24)$ & & 662 & 769 & 873 & 956 & 1007 & 1041 \\
\hline
\end{tabular}

$z<0$ (resp. $z>0$ ) experience positive (resp. negative) axial strain $\epsilon_{11}$. The maximum strain is reached when the curvature is maximum, i.e. for loading condition B of Fig. $1 \mathrm{~b}$ ). During the unloading stage (BC), the strain is slightly decreasing while the strain magnitude is strongly reduced in the reverse bending phase (CD). Nevertheless, due to plasticity development and the presence of the peel force, a residual strain remains in the peel arm. This residual strain is homogeneous in the thickness, meaning that the curvature is close to $K_{D}=0$ (straight arm at the end). It is also interesting to mention that for the condition presented here $\left(\Phi=90^{\circ}\right)$, the strain profile in the thickness is almost skewsymmetric. This is in close agreement with the bending theory of the beam which is based on the assumption that any material point entering into the process zone will experience pure bending/reverse bending with the axial strain of the form $\epsilon_{11}=-K z$. Fig. $7 \mathrm{~b}$ ) presents the strain development inside the film thickness at the exact end of the bending process, condition B. Since $K_{B}$ 
can be evaluated from finite element simulations, the theoretical predictions of the axial strain given by $\epsilon_{11}=-K_{B} z$ in the cross-section is also added to the figure. We observe that for a peel angle of $\Phi=90^{\circ}$ where $K_{B}=3759 m^{-1}$ (i.e. $R=248 \mu m$ ), a very good agreement exists between the theory and the FE results. A similar comparison is made for two other angles : $\Phi=60^{\circ}$ and $\Phi=135^{\circ}$. For $\Phi=135^{\circ}$, it is seen that the strain distribution is slightly nonlinear, with a larger absolute value of the axial strain at the bottom surface $(z<0)$ when compared to the top surface $(z>0)$. In that configuration the curvature is $K_{B}=6468 m^{-1}$ corresponding to a radius of $R=137 \mu m$. It will be shown next that the limited correlation of the present theory in reproducing the expected value of the interface fracture energy for a large $\Phi$ angle is partially due to the small radius observed. This conclusion was already drawn in Aravas et al. (1989). Indeed, the plane strain bending approach (with $\epsilon_{11}=-K z$ ) is considered by Hill (1950) as a good approximation assuming that the minimum radius is larger than four to five times the film thickness. With $t=35 \mu \mathrm{m}$ and $\Phi=135^{\circ}, R$ is smaller than $4 t$. For $\Phi=60^{\circ}$, the measured value of the curvature obtained by FE simulations is $K_{B}=2434 m^{-1}(R=393 \mu m)$. It is observed in Fig. $7 \mathrm{~b}$ ), that the axial strain $\epsilon_{11}$ vanishes for $z=5 \mu \mathrm{m}$ in the FE calculation while in the theory of pure bending, the condition is fulfilled for the central surface $(z=0)$. This $\mathrm{FE}$ result $\left(\epsilon_{11}=0\right.$ for $\left.z \neq 0\right)$ observed mostly for $\Phi<90^{\circ}$ is due to the fact that instead of pure bending, a more complex loading path prevails with bending and extension. For $\Phi<90^{\circ}$, the peel force is significantly larger than for $\Phi>90^{\circ}$, see Rivlin (1944) or Williams and Kauzlarich (2005).

Fig. 8 presents the stress-strain loading path faced by the six material points defined in Fig. 7 a) during the peeling process for two angles : $\Phi=$ $90^{\circ}$ and $\Phi=135^{\circ}$. The loading stages (OABCD) are also added in Fig. 8 


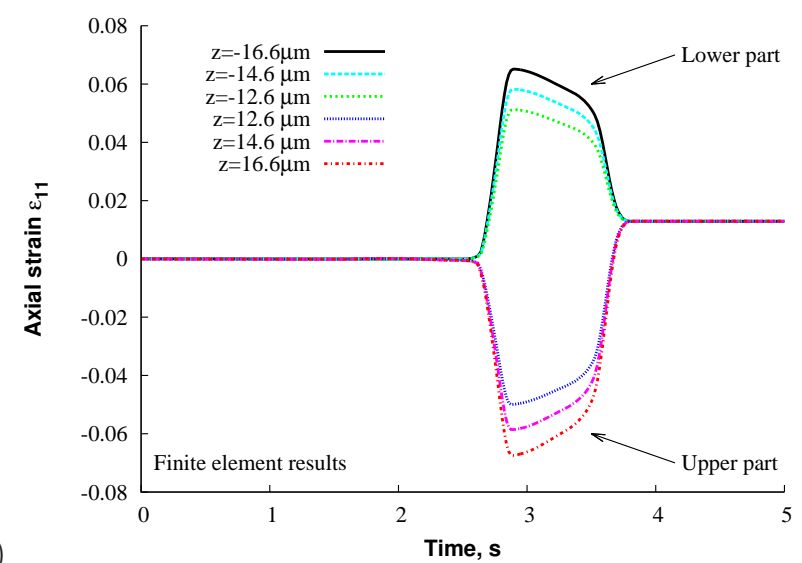

a)

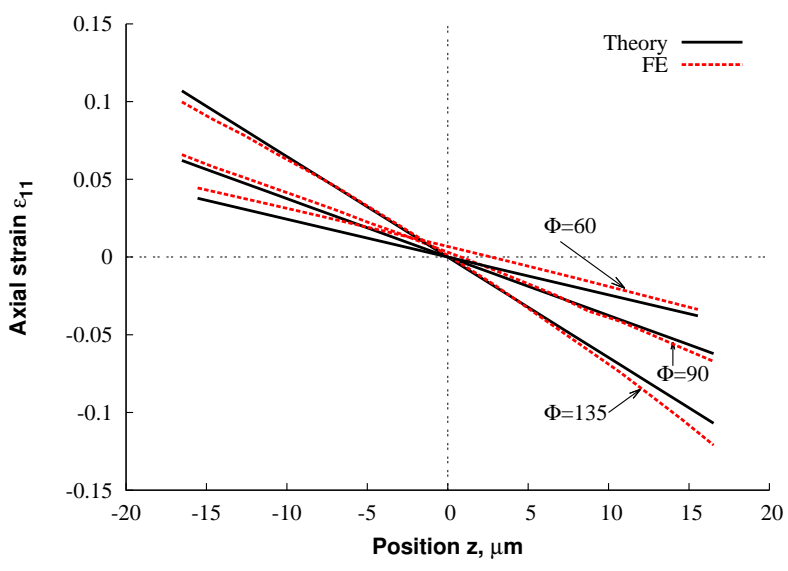

Figure 7: a) Time evolution of the axial strain component $\epsilon_{11}$ within the film thickness for the peel angle $\Phi=90^{\circ}$. Predictions are obtained by finite element calculations. b) Maximum axial strain $\epsilon_{11}$ within the film cross-section captured by the finite element calculations. A comparison is made between FE results and the bending approach for three peel angles : $60^{\circ}, 90^{\circ}$ and $135^{\circ}$. Material parameters are those of Table 1 with $\gamma=40$.

a). Material parameters listed in Table 1 with $\gamma=40$ are adopted in the calculations. Fig. 8 a) shows that for an angle $\Phi=90^{\circ}$, the assumption of pure bending/reverse bending is mostly satisfied. During the loading path (OAB), the trajectory of all points in the stress-strain plane is identical up to a maximum axial strain $\epsilon_{11}^{\max }=-K_{B} z$. For material points located close to the external 
surface of the film, the accumulated strain $\bar{\epsilon}$ up to point B is large enough so that the yield stress is close to its saturation value $\sigma_{o}+Q$. Therefore, during the reverse bending process $(\mathrm{CD})$, the stress level remains almost constant. In that stage, the material behavior can be approximated by an elastic-perfectly plastic response. For $\Phi=135^{\circ}$ (see Fig. 8 b), the same conclusions hold except for the maximum strain. Indeed, we observe that the maximum axial strain at the top surface is $20 \%$ larger in absolute value than the strain experienced at the bottom surface. From the knowledge of the curvature $K_{B}$, the theoretical prediction of the stress $\sigma_{11}$ versus strain $\epsilon_{11}$ response is also added to Figs 8 a) and b) for two material points located at $z= \pm 16.6 \mu m$ (see dotted lines with squares or circles). It is clearly observed that some discrepancies exist between the theory and the finite element response. Of course, in the theory, the distribution of axial strain is assumed skew-symmetric, which is not clearly the case in finite element simulations. The pure bending assumption is better fulfilled for $\Phi=90^{\circ}$ than for $\Phi=135^{\circ}$.

At least for the parameters adopted (as indicated in Table 1), the proposed expression Eq. (21) for the work done by bending plasticity enables the recovery precisely of the interface fracture energy adopted in the numerical calculations when the peel angle is $\Phi=90^{\circ}$, as presented in Table 2. Peel tests with an angle $\Phi$ in the range $\left[+45^{\circ},+135^{\circ}\right]$ are also simulated. The reference parameters of Table 1 are still adopted with $\gamma=40$. Fig. 9 displays the estimated value of the interface fracture energy $\Gamma$ assuming that the force per unit width $P$ and the maximum curvature $K_{B}$ are known quantities (obtained from FE simulations). As already mentioned, the pure bending theory predicts a consistent interface fracture energy using Eqs (19) and (21) when $\Phi=90^{\circ}$. It is observed that for $\Phi=120^{\circ}, 135^{\circ}$, the proposed model based on the expression of $\Psi$ leads to an 


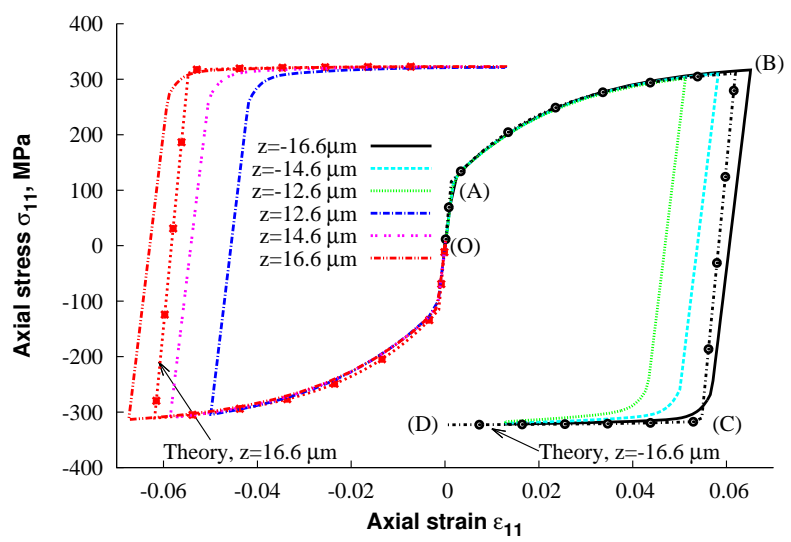

a)

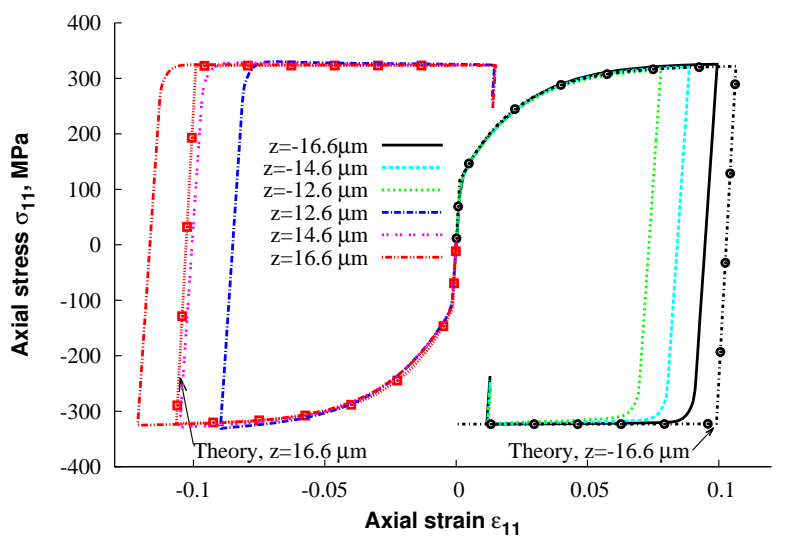

b)

Figure 8: Loading path faced by material points located in the film cross-section during the peeling process. a) Peel angle of $90^{\circ}$, b) peel angle of $135^{\circ}$. A local orientation is adopted in the finite element simulations. The reference parameters are those of Table 1 with $\gamma=40$.

estimate for $\Gamma$ of $1160 \mathrm{~N} / \mathrm{m}$, instead of $1055 \mathrm{~N} / \mathrm{m}$. Clearly, the film radius is too small when compared to the film thickness for the bending theory to be accurate, see Hill (1950). For $\Phi=45^{\circ}, 60^{\circ}$, the strain map in the film thickness is not fully consistent with a pure bending approach, see Fig. 7 b). Indeed, for $\Phi<90^{\circ}$, the stretching of the film is more intense, and the tensile contribution is noticeable. It is observed that for $\Phi=60^{\circ}$, the estimate of $\Gamma$ is still accurate 
(around $5 \%$ difference, $\Gamma(60)=990 \mathrm{~N} / \mathrm{m})$. The situation is different for $\Phi=45^{\circ}$ with more than $10 \%$ difference $(\Gamma(45)=909 N / m)$. The prediction of $\Gamma$ based on the approximate relation $(23)$ is not presented since it coincides almost with the one given by Eq. (21). The prediction of $\Gamma$ based on the elastic-perfectly plastic approach Eq. (24) is also superimposed to Fig. 9. The elastic-perfectly plastic approach is underestimating $\Gamma$ for the considered material parameters, except for the large angle condition $\left(\Phi=135^{\circ}\right.$ or $\left.\Phi=120^{\circ}\right)$. So from Fig. 9, it can be mentioned that the value of the interface fracture energy can be reasonably captured by the present work, from the knowledge of the experimental force and curvature for tests with peel angle around $\Phi=90^{\circ}$. Interestingly, it is seen that the closed form expression derived from Eq. (23) also provides consistent estimate and can be used instead of Eq. (21), at least for the material parameters considered here.

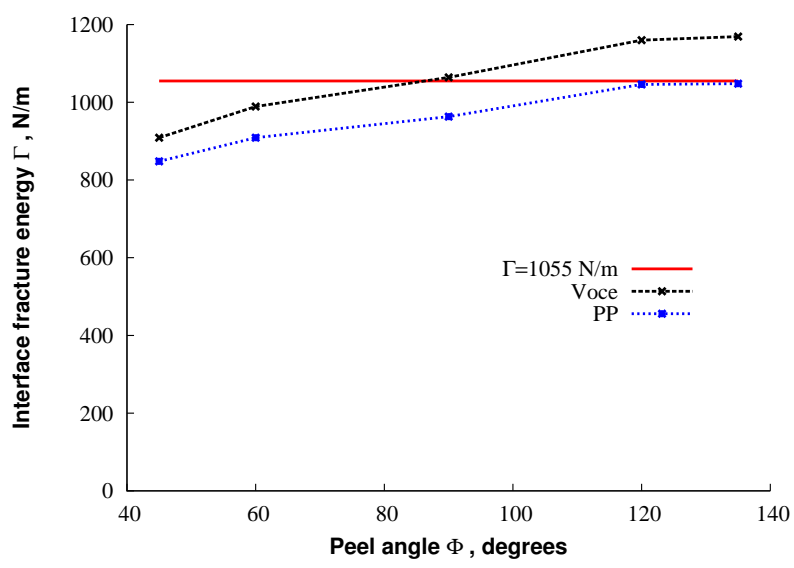

Figure 9: Effect of the peel angle $\Phi$ on the estimated interface fracture energy $\Gamma$. The predictions based on the present work Eq. (21) and on Eq. (24) derived for an elastic-perfectly plastic material are compared. The reference parameters are listed in Table 1 with $\gamma=40$.

From previous works of the literature (Wei and Hutchinson (1998), Kim and 
Kim (1988), Wei (2004), Martiny et al. (2008) or Thouless and Yang (2008)), it is known that the results of finite element simulations of peel tests for an elastic-plastic film are highly influenced by the properties of the interface, $i . e$. the parameters of the cohesive law (here the interface fracture energy $\Gamma$ and the peak stress $\hat{\sigma}$ ). Next, we propose to investigate their relative influence on the force and curvature during a peel test. The material behavior is still described by a Voce law with parameters listed in Table 1 and $\gamma=40$. We assume that for a given peel angle $\Phi=90^{\circ}$, the peel force $P$ is known. For different values of $\Gamma$, the peak stress $\hat{\sigma}$ is adjusted so that the peel force $P$ at $\Phi=90^{\circ}$ is unchanged, see Martiny et al. (2008). Fig. 10 displays the evolution of the peel force $P$ and the curvature $K_{B}$ for 6 sets of parameters $(\Gamma, \hat{\sigma})$ defined in Table 3. As $\Gamma$ is reduced, the peak stress $\hat{\sigma}$ takes larger value leading to a larger curvature (smaller radius $R$ ). Therefore the work done by bending plasticity is enlarged. For $\Gamma=1055 \mathrm{~N} / \mathrm{m}$ and $\hat{\sigma}=10 \mathrm{MPa}$, the finite element simulation of the peel test at $\Phi=90^{\circ}$ has provided a curvature of $K_{B}=3759 m^{-1}(R=248 \mu m)$ while for $\Gamma=527.5 \mathrm{~N} / \mathrm{m}$ and $\hat{\sigma}=72 \mathrm{MPa}$, the curvature is $K_{B}=6042 \mathrm{~m}^{-1}$ corresponding to a radius of $R=148 \mu \mathrm{m}$. After this calibration, the peel force $P$ is evaluated for four additional angles $\Phi=45^{\circ}, 60^{\circ}, 120^{\circ}$ and $135^{\circ}$. Fig. 10 shows that the peel force is quite insensitive to the 6 sets $(\Gamma, \hat{\sigma})$ for $\Phi>90^{\circ}$ while it varies significantly when $\Phi<90^{\circ}$. This effect is larger for $\Phi=45^{\circ}$ than for $\Phi=60^{\circ}$. For instance, with $\Phi=45^{\circ}$, one has $P=3777 \mathrm{~N} / \mathrm{m}$ for $\Gamma=1055 \mathrm{~N} / \mathrm{m}$ and $P=3300 \mathrm{~N} / \mathrm{m}$ when the interface fracture energy is reduced by a factor of two: $\Gamma=527.5 \mathrm{~N} / \mathrm{m}$. Remember that $\hat{\sigma}$ is adjusted in order to preserve the peel force level at $\Phi=90^{\circ}$. Fig. 10 indicates also that the curvature is the most sensitive feature to any variation of $(\Gamma, \hat{\sigma})$, see also Thouless and Yang (2008) for the role of the peak stress on the plasticity development at the onset of the crack propagation. Therefore, to identify the interface fracture energy $\Gamma$, a peel 
test at $\Phi=90^{\circ}$ where the force and curvature are recorded, may be sufficient. Nevertheless, it seems also important to validate this estimate by confirming the prediction of the force by performing another test; a peel test at angle $\Phi=45^{\circ}$ seems to be a good candidate. Indeed, the peel force $P$ for small angle $\Phi$ is quite sensitive to the value of the interface fracture energy. Of course, all the present results are valid for the specific cohesive law Eq. (27) and for the given material behavior.

Table 3: Set $(\Gamma, \hat{\sigma})$ leading to the same peel force per unit width for the peel angle $\Phi=90^{\circ}$.

\begin{tabular}{|c|c|c|c|c|c|c|}
\hline$\Gamma[\mathrm{N} / \mathrm{m}]$ & 1055 & 949.5 & 844 & 738.5 & 633 & 527.5 \\
\hline$\hat{\sigma}[\mathrm{MPa}]$ & 10 & 15 & 21 & 31 & 46 & 72 \\
\hline
\end{tabular}

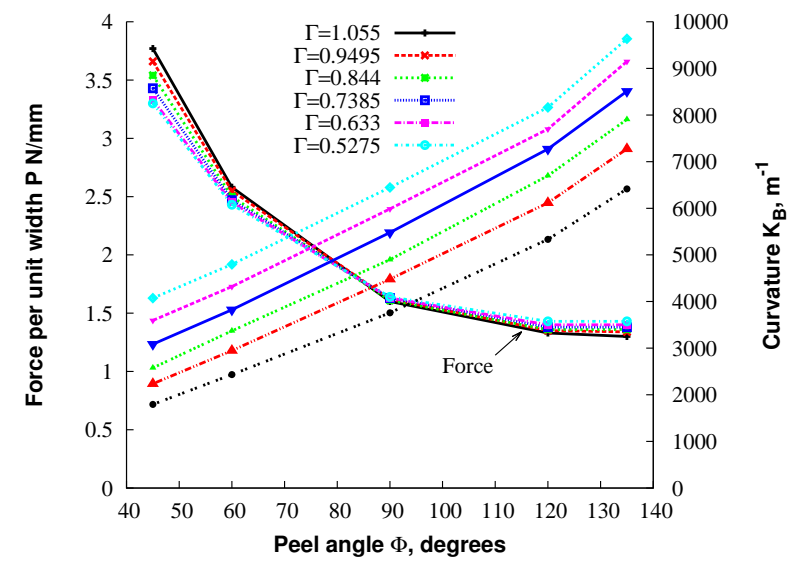

Figure 10: Force per unit width and curvature for various peel angles $\Phi$. Note that the cohesive law parameters listed in Table 3 are adjusted so that the level of the peel force $P\left(\Phi=90^{\circ}\right)$ is preserved for all angles.

\section{Conclusion}

A semi-analytical expression for the work done by bending plasticity is derived for a material presenting a Voce hardening law. The proposed expression 
contains two situations addressed in the literature where the film has an elasticperfectly plastic behavior, Kim and Kim (1988) or a bilinear response, Kinloch et al. (1994). The work done by bending plasticity is highly sensitive to the film curvature. As proposed previously in the literature for other material constitutive laws, the knowledge of this work enables the extraction of the interface fracture energy from the peel force measurement. By comparison with finite element calculations performed for various angles in the range of $\left[45^{\circ}, 135^{\circ}\right]$, it is found that the present theory is accurate to analyze the peel test for $\Phi=90^{\circ}$ and provides a satisfactory estimate of the interface fracture energy for other peel angles. For $\Phi=90^{\circ}$, at least for the material parameters adopted in the present paper, the assumption of pure bending in the process zone is a satisfactory hypothesis. It is also observed that depending on the material parameters, the elastic-perfectly plastic approach proposed by Kim and Kim (1988), Aravas et al. (1989) can also be justified even for a Voce type material when the saturation of the yield stress is observed for limited strain.

The cohesive law parameters (interface fracture energy and peak stress) can be estimated by an inverse method, assuming that the force and curvature have been identified with recourse to finite element simulations and/or experiments. The identification of the cohesive law parameters is usually done by considering a $90^{\circ}$ peel test but could benefit from a further validation based on peel tests carried out for small peel angle $\Phi<90^{\circ}$.

\section{Acknowledgments}

The authors acknowledge the support of Agence Nationale de Recherche through the program Labcom LEMCI ANR-14-LAB7-0003-01.

The research leading to these results has received funding from the European Union's Horizon 2020 research and innovation programme (Excellent Science, 
Marie Sklodowska-Curie Actions) under REA grant agreement 675602 (OUTCOME project).

\section{A Derivation of the expression for the work done by bending plasticity}

The present appendix provides some details concerning the derivation of the expression for the work done by bending plasticity $\Psi$ along the loading path (OABCD). From the elastic response of the film along path (OA), as depicted in Fig. 1 b), one obtains based on Eq. (6):

$$
\int_{0}^{K_{e}} M d K=\frac{\sigma_{o}^{2} t}{6 E}
$$

Since for a thin copper film, the curvature $K_{B}$ is larger than $K_{e}$, one needs to evaluate the contribution to $\Psi$ along $(\mathrm{AB})$. The relationship between the moment $M$ and the curvature $K$ is given by Eq. (10). With the definition $F\left(-\frac{2 \gamma K}{\sqrt{3}}, \frac{t}{2}\right)=-\exp \left(-\frac{\gamma K t}{\sqrt{3}}\right)\left(\frac{t \sqrt{3}}{4 \gamma K}+\frac{3}{4 \gamma^{2} K^{2}}\right)$, and with a change of variable $u=$ $\frac{\gamma K t}{\sqrt{3}}$, one easily gets:

$$
\int_{K_{e}}^{K_{B}} F\left(-\frac{2 \gamma K}{\sqrt{3}}, \frac{t}{2}\right) d K=\frac{3}{4 \gamma^{2}}\left[G\left(-\frac{\gamma t}{\sqrt{3}}, K_{B}\right)-G\left(-\frac{\gamma t}{\sqrt{3}}, K_{e}\right)\right]
$$

where $G(x, y)=\frac{\exp (x y)}{y}$. The contribution linked to the term $F\left(-\frac{2 \gamma K}{\sqrt{3}}, h\right)$ with $h=\frac{\sigma_{o} \sqrt{3}}{2 E K}$ is evaluated in a straightforward manner, so the work done by bending 
plasticity along the path $(\mathrm{AB})$ is:

$$
\begin{aligned}
\int_{K_{e}}^{K_{B}} M d K= & \frac{\alpha t^{2}}{2 \sqrt{3}}\left(K_{B}-K_{e}\right)+\left(\frac{\sigma_{o}}{\sqrt{3}}-\frac{\sqrt{3} \alpha}{2}\right) \frac{\sigma_{o}^{2}}{E^{2}}\left(\frac{1}{K_{e}}-\frac{1}{K_{B}}\right) \\
& -\frac{\sqrt{3} \beta}{\gamma^{2}}\left[G\left(-\frac{\gamma t}{\sqrt{3}}, K_{B}\right)-G\left(-\frac{\gamma t}{\sqrt{3}}, K_{e}\right)\right] \\
& +\frac{\sqrt{3} \beta}{\gamma} \exp \left(-\frac{\gamma \sigma_{o}}{E}\right)\left(\frac{\sigma_{o}}{E}+\frac{1}{\gamma}\right)\left(\frac{1}{K_{B}}-\frac{1}{K_{e}}\right)
\end{aligned}
$$

For the unloading path (BC), the contribution to $\Psi$ is readily obtained:

$$
\int_{K_{B}}^{K_{B}-K_{B}^{*}} M d K=-M_{B} K_{B}^{*}+\frac{E t^{3}}{18}\left(K_{B}^{*}\right)^{2}
$$

The last term corresponding to the reverse plastic loading phase (CD) is the most complex contribution. Indeed, the expression of $M$ is given by Eq. (15) where an integral term is already present. In addition, it is related to the position of the elastic-plastic transition surface $h^{\prime}$ defined by an implicit relation (13). Therefore one can simply notice that:

$$
\begin{array}{r}
\int_{K_{B}-K_{B}^{*}}^{0} M d K=\left[\frac{8 E K_{B} h_{B}^{3}}{9}-\frac{2 \alpha}{\sqrt{3}}\left(h_{B}^{2}+\frac{t^{2}}{4}\right)\right. \\
\left.+\frac{4 \beta}{\sqrt{3}} F\left(-\frac{2 \gamma K_{B}}{\sqrt{3}}, h_{B}\right)\right]\left(K_{B}^{*}-K_{B}\right)+I
\end{array}
$$

where the term $I$ is defined by Eq. (22). As mentioned in Section 2, for a metallic film with large values of $K_{B}$ and $\gamma$, the accumulated strain Eq. (14) (resp. $h^{\prime}$ ) can be approximated as proposed in Eq. (16) (resp. by $\left.h^{\prime}=\frac{\sqrt{3} \alpha}{E\left(K_{B}-K\right)}\right)$. After a standard mathematical development, the integral term $I$ is evaluated and the approximate closed form expression Eq. (23) is found. 


\section{References}

ABAQUS (2013) Abaqus v6.13 User's Manual, version 6.13 edn. ABAQUS Inc., Richmond, USA

Aravas N, Kim K, Loukis M (1989) On the mechanics of adhesion testing of flexible films. Materials Science and Engineering, A 107:159-168

Girard G, Jrad M, Bahi S, Martiny M, Mercier S, Bodin L, Nevo D, Dareys S (2018) Experimental and numerical characterization of thin woven composites used in printed circuit boards for high frequency applications. Composite structures 193:140 - 153

Hill R (1950) The mathematical theory of plasticity. Oxford University Press, Oxford

IPC (1994) Peel strength of metallic clad laminates, IPC-TM-650 2.4.8, Institute for Interconnecting and Packaging Electronic Circuits, www.ipc.org/TM/2. $4.8 c \cdot p d f$

Kendall K (1973) Shrinkage and peel strength of adhesive joints. Journal of Physics D: Applied Physics 6(15):1782-1787

Kendall K (1975) Thin-film peeling-the elastic term. Journal of Physics D: Applied Physics 8(13):1449-1452

Kim J, Kim K, Kim Y (1989) Mechanical effects in peel adhesion test. J Adhesion Sci Technol 3:175-187

Kim K, Aravas N (1988) Elastoplastic analysis of the peel test. International Journal of Solids and Structures 24:417-435

Kim K, Kim J (1988) Elasto-plastic analysis of the peel test for thin film adhesion. Transactions of the ASME 110:266-273 
Kinloch A, Lau C, JG W (1994) The peeling of flexible laminates. International Journal of Fracture 66:45-70

Martiny P, Lani F, Kinloch A, Pardoen T (2008) Numerical analysis of the energy contributions in peel tests : a steady-state multilevel finite element approach. International Journal of Adhesion and Adhesives 28:222-236

Moidu K, Sinclair A, Spelt J (1998) On the determination of fracture energy using the peel test. Journal of Testing and Evaluation 26:247-254

Molinari A, Ravichandran G (2008) Peeling of elastic tapes: Effects of large deformations, pre-straining, and of a peel-zone model. The Journal of Adhesion 84(12):961-995

Ortiz M., Pandolfi A. (1999) Finite-deformation irreversible cohesive elements for three-dimensional crack-propagation analysis. International journal for numerical methods in engineering 44:1267-1282

Rivlin R (1944) The effective work of adhesion. Paint Technology 9:215-218

Song J, Yu J (2002) Analysis of T-peel strength in a Cu/Cr/Polyimide system. Acta Materialia 50:3985-3994

Thouless MD, Yang QD (2008) A parametric study of the peel test. International Journal of Adhesion and Adhesives 28:176-184

Tvergaard V, Hutchinson J (1993) The influence of plasticity on mixed mode interface toughness. Journal of the Mechanics and Physics of Solids 41:11191135

Voce E (1948) The relationship between stress and strain for homogeneous deformations. J Inst Metals 74:537-562 
Wei Y (2004) Modeling non linear peeling of ductile thin films - critical assessment of analytical bending models using FE simulations. International Journal of Solids and Structures 41:5087-5104

Wei Y, Hutchinson J (1998) Interface strength, work of adhesion and plasticity in peel test. International Journal of Fracture 93:315-333

Wei Y, Zhao H (2008) Peeling experiments of ductile thin films along ceramic substrates - critical assessment of analytical models. International Journal of Solids and Structures 45:3779-3792

Williams J, Kauzlarich J (2005) The influence of peel angle on the mechanics of peeling flexible adherends with arbitrary load - extension characteristics. Tribology International 38:951-958

Yang QD,Thouless MD, Ward SW (2000) Analysis of the symmetrical $90^{\circ}$ - peel test with extensive plastic deformation. Journal of Adhesion 72:115-132

Yang QD,Thouless MD (2001) Mixed-mode fracture analyses of plasticallydeforming adhesive joints. International Journal of Fracture 110:175-187

Zhao H, Wei Y (2007) Determination of interface properties between micronthick metal film and ceramic substrate using peel test. International Journal of Fracture 144:103-112 